PROCEEDINGS OF THE

AMERICAN MATHEMATICAL SOCIETY

Volume 137, Number 4, April 2009, Pages 1205-121

S 0002-9939(08)09768-2

Article electronically published on November 21, 2008

\title{
VANISHING OF MODULAR FORMS AT INFINITY
}

\author{
SCOTT AHLGREN, NADIA MASRI, AND JEREMY ROUSE
}

(Communicated by Wen-Ching Winnie Li)

\begin{abstract}
We give upper bounds for the maximal order of vanishing at $\infty$ of a modular form or cusp form of weight $k$ on $\Gamma_{0}(N p)$ when $p \nmid N$ is prime. The results improve the upper bound given by the classical valence formula and the bound (in characteristic $p$ ) given by a theorem of Sturm. In many cases the bounds are sharp. As a corollary, we obtain a necessary condition for the existence of a non-zero form $f \in S_{2}\left(\Gamma_{0}(N p)\right)$ with $\operatorname{ord}_{\infty}(f)$ larger than the genus of $X_{0}(N p)$. In particular, this gives a (non-geometric) proof of a theorem of Ogg, which asserts that $\infty$ is not a Weierstrass point on $X_{0}(N p)$ if $p \nmid N$ and $X_{0}(N)$ has genus zero.
\end{abstract}

\section{IntRoduction AND STATEMENT OF RESUltS}

Let $M_{k}\left(\Gamma_{0}(N)\right)$ denote the complex vector space of holomorphic modular forms of weight $k$ and level $N$, and let $S_{k}\left(\Gamma_{0}(N)\right.$ ) denote the subspace of cusp forms (see, for example, 4 for background). If $f(z)$ is a non-zero element of $M_{k}\left(\Gamma_{0}(N)\right.$ ), and $q:=e^{2 \pi i z}$, then $f$ has a Fourier expansion at $\infty$ of the form

$$
f(z)=\sum_{n=n_{0}}^{\infty} a(n) q^{n} \quad \text { with } a\left(n_{0}\right) \neq 0 .
$$

Given such a form $f$, we define

$$
\operatorname{ord}_{\infty}(f):=n_{0} .
$$

The following question is very natural:

For a non-zero element $f \in M_{k}\left(\Gamma_{0}(N)\right)$ (respectively $S_{k}\left(\Gamma_{0}(N)\right)$ ), what is the largest possible value of $\operatorname{ord}_{\infty}(f)$ ?

For convenience, we define $\Gamma:=\mathrm{SL}_{2}(\mathbb{Z})$. By the valence formula, we know that the total number of zeros of a non-zero element $f \in M_{k}\left(\Gamma_{0}(N)\right.$ ) (counted in local coordinates in the usual way), is given by $\frac{k}{12}\left[\Gamma: \Gamma_{0}(N)\right]$ (see, for example, Chapter $\mathrm{V}$ of [12]). An element of $M_{k}\left(\Gamma_{0}(N)\right)$ may (depending on the values of $N$ and $k$ ) have forced vanishing at elliptic points. We denote by $\alpha(N, k)$ the number of zeros forced by this consideration, and by $\epsilon_{\infty}(N)$ the number of cusps of $\Gamma_{0}(N)$ (see (3.2),

Received by the editors April 9, 2008.

2000 Mathematics Subject Classification. Primary 11F11, 11F33, $14 \mathrm{H} 55$.

The first author thanks the National Science Foundation for its support through grant DMS 01-34577.

(C)2008 American Mathematical Society Reverts to public domain 28 years from publication 
(3.4) for the precise definitions). Then we have

$$
\begin{aligned}
& 0 \neq f \in M_{k}\left(\Gamma_{0}(N)\right) \Longrightarrow \operatorname{ord}_{\infty}(f) \leq \frac{k}{12}\left[\Gamma: \Gamma_{0}(N)\right]-\alpha(N, k), \\
& 0 \neq f \in S_{k}\left(\Gamma_{0}(N)\right) \Longrightarrow \operatorname{ord}_{\infty}(f) \leq \frac{k}{12}\left[\Gamma: \Gamma_{0}(N)\right]-\alpha(N, k)-\epsilon_{\infty}(N)+1 .
\end{aligned}
$$

On the other hand, each of the spaces $M_{k}\left(\Gamma_{0}(N)\right)$ and $S_{k}\left(\Gamma_{0}(N)\right)$ has a basis consisting of forms with rational coefficients. Using this basis, one can construct an integral basis in "echelon form." To be precise, let $d$ denote the dimension of the space in question. Then we have a basis of forms $\left\{f_{1}, \ldots, f_{d}\right\}$ with integer coefficients and with the property that

$$
\begin{array}{cc}
f_{1}(z) & =a_{1} q^{c_{1}}+O\left(q^{c_{1}+1}\right), \\
f_{2}(z) & =a_{2} q^{c_{2}}+O\left(q^{c_{2}+1}\right), \\
\vdots & \vdots \\
f_{d}(z)= & a_{d} q^{c_{d}}+O\left(q^{c_{d}+1}\right) .
\end{array}
$$

Here each leading coefficient $a_{i}$ is a non-zero integer, and $c_{1}<c_{2}<\cdots<c_{d}$. It is clear that the maximal order of vanishing at infinity of any non-zero form in the space is equal to $c_{d}$.

Denote the maximal order of vanishing of any non-zero form in $M_{k}\left(\Gamma_{0}(N)\right)$ by $m_{N, k}$ and the maximal order of vanishing of any non-zero form in $S_{k}\left(\Gamma_{0}(N)\right)$ by $s_{N, k}$. Using (1.1) and the above basis, we see that

$$
\begin{aligned}
\operatorname{dim}\left(M_{k}\left(\Gamma_{0}(N)\right)\right)-1 & \leq m_{N, k} \leq \frac{k}{12}\left[\Gamma: \Gamma_{0}(N)\right]-\alpha(N, k), \\
\operatorname{dim}\left(S_{k}\left(\Gamma_{0}(N)\right)\right) \leq s_{N, k} & \leq \frac{k}{12}\left[\Gamma: \Gamma_{0}(N)\right]-\alpha(N, k)-\epsilon_{\infty}(N)+1 .
\end{aligned}
$$

It is possible to construct examples of spaces for which $m_{N, k}$ (respectively $s_{N, k}$ ) falls at either end of the allowable range. However, the exact value of these quantities is in general mysterious. For example, it is conjectured that if $N$ is squarefree, then $\infty$ is not a Weierstrass point on the modular curve $X_{0}(N)$. Letting $g(N)$ denote the genus of $X_{0}(N)$, this is equivalent to the assertion that $s_{N, 2}=\operatorname{dim}\left(S_{2}\left(\Gamma_{0}(N)\right)\right)=g(N)$ for such $N$. This has been verified by William Stein for squarefree $N \leq 3223$. On the other hand, Lehner and Newman [9] and Atkin [1] proved that $s_{N, 2}>\operatorname{dim}\left(S_{2}\left(\Gamma_{0}(N)\right)\right)$ for many families of $N$ which are not squarefree.

Using geometric arguments in characteristic $p$, Ogg [10] proved that $\infty$ is not a Weierstrass point on $X_{0}(N p)$ whenever $p \nmid N$ is prime and $X_{0}(N)$ has genus zero. Recently, Kohnen 8 and Kilger [7] have used techniques from the theory of modular forms mod $p$ to reprove Ogg's result for certain curves $X_{0}(p \ell)$ when $p$ and $\ell$ are distinct primes. As a corollary to our first theorem, we obtain a proof of Ogg's result which uses only standard facts from the theory of modular forms mod $p$.

To state the first result, when $p \| N$ we require the Atkin-Lehner involution $W_{p}^{N}$ on $S_{2}\left(\Gamma_{0}(N)\right.$ ) (see (3.5) below). For a power series $f=\sum a(n) q^{n}$ with rational coefficients and bounded denominators, we recall that $v_{p}(f):=\inf \left\{v_{p}(a(n))\right\}$. Then we have the following, which was proved for certain $N$ of the form $p \ell$ by Kohnen and Kilger. 
Theorem 1.1. Suppose that $p \geq 5$ is a prime with $p \| N$ and that $f \in S_{2}\left(\Gamma_{0}(N)\right) \cap$ $\mathbb{Q}[[q]]$ has $v_{p}(f)=0$ and $v_{p}\left(f \mid W_{p}^{N}\right) \geq 0$. Then $\operatorname{ord}_{\infty}(f) \leq g(N)$.

As an easy corollary, we obtain Ogg's result.

Corollary 1.2. If $p$ is a prime with $p \| N$, and $g(N / p)=0$, then $\infty$ is not a Weierstrass point on $X_{0}(N)$.

We now state the results for general weights. If $f \in M_{k}\left(\Gamma_{0}(N)\right)$, then let $\alpha_{2}(N, k)$ and $\alpha_{3}(N, k)$ denote the number of complex zeros of $f$ which are forced at the elliptic points of orders 2 and 3 (see (3.3) for the precise values). We will consider levels $N$ of the form $N=p N^{\prime}$ where $p \geq 5$ is a prime with $p \nmid N^{\prime}$. For such an $N$, and for weights $k$ which are sufficiently small relative to $p$, we obtain an improvement of the upper bounds in (1.3) for each of the quantities $m_{N, k}$ and $s_{N, k}$. We note that Theorem 4.2 gives a more precise statement for any particular form $f$.

Theorem 1.3. Suppose that $k \geq 2$, that $p \geq k+3$ is prime, and that $N$ is an integer with $p \| N$. Suppose that $f(z) \in M_{k}\left(\Gamma_{0}(N)\right)$ and that $f \neq 0$. Then we have

$$
\operatorname{ord}_{\infty}(f) \leq \frac{k p}{12} \cdot\left[\Gamma: \Gamma_{0}(N / p)\right]-\frac{1}{2} \alpha_{2}(N / p, k p)-\frac{1}{3} \alpha_{3}(N / p, k p) .
$$

Theorem 1.4. Suppose that $k \geq 2$, that $p \geq \max (5, k+1)$ is prime, and that $N$ is an integer with $p \| N$. Suppose that $f(z) \in S_{k}\left(\Gamma_{0}(N)\right)$ and that $f \neq 0$. Then we have

$$
\operatorname{ord}_{\infty}(f) \leq \frac{k p}{12} \cdot\left[\Gamma: \Gamma_{0}(N / p)\right]-\frac{1}{2} \alpha_{2}(N / p, k p)-\frac{1}{3} \alpha_{3}(N / p, k p)-\epsilon_{\infty}(N / p)+1 .
$$

The bounds in these results are sharp for many spaces of forms. Let $\eta(z)$ be the usual Dedekind eta-function, defined by

$$
\eta(z):=q^{\frac{1}{24}} \prod_{n=1}^{\infty}\left(1-q^{n}\right) .
$$

For one family of examples, define $f(z) \in M_{4}\left(\Gamma_{0}(15)\right)$ by

$$
f(z):=\frac{\eta(z) \cdot \eta^{15}(15 z)}{\eta^{3}(3 z) \cdot \eta^{5}(5 z)}=q^{8}+\cdots,
$$

and if $p>5$ is prime, then define $g(z) \in M_{4}\left(\Gamma_{0}(15 p)\right)$ by

$$
g(z):=f(p z)=q^{8 p}+\cdots \in M_{4}\left(\Gamma_{0}(15 p)\right) .
$$

We have $\alpha(15 p, 4)=0$, so in this case the upper bound in (1.3) is $\frac{4}{12}\left[\Gamma: \Gamma_{0}(15 p)\right]=$ $8 p+8$. We see that the actual order of vanishing matches the bound $\frac{4 p}{12}\left[\Gamma: \Gamma_{0}(15)\right]=$ $8 p$ provided by Theorem 1.3. Infinite families of related examples will be discussed in the last section.

We also remark that the hypothesis on the size of $p$ is necessary. For example, in the space $M_{6}\left(\Gamma_{0}(35)\right)$, there is a form whose $q$-expansion begins with $q^{21}+\cdots$. On the other hand, we have $\frac{6 \cdot 7}{12}\left[\Gamma: \Gamma_{0}(5)\right]-\frac{1}{2} \alpha_{2}(5,42)=20$, from which we see that the conclusion does not hold when $p=7$.

To see that Theorem 1.4 is sharp, consider the space $S_{4}\left(\Gamma_{0}(60)\right)$. We have $\alpha(60,4)=0$ and $\epsilon_{\infty}(60)=12$, so the upper bound provided by (1.3) is 37 . On the other hand, we have $\left[\Gamma: \Gamma_{0}(12)\right]=24$ and $\epsilon_{\infty}(12)=6$, so the bound in Theorem 1.4 is $\frac{4 \cdot 5}{12} \cdot 24-5=35$. In fact, there is a form in this space whose $q$-expansion is $q^{35}+\cdots$. More examples will be provided in the last section. Again, the assumption on $p$ is 
necessary; to see this note that there is a form $f \in S_{8}\left(\Gamma_{0}(35)\right)$ whose $q$-expansion is $f=q^{26}+\cdots$.

A computation using (1.1) shows that $s_{N, k}$ attains values in an interval, considered asymptotically with respect to $N$, of length $\frac{p+1}{12}\left[\Gamma: \Gamma_{0}(N / p)\right]$. Theorem 1.4 implies that $s_{N, k}$ lies in the narrower range

$$
\begin{aligned}
\operatorname{dim}\left(S_{k}\left(\Gamma_{0}(N)\right) \leq s_{N, k} \leq \frac{k p}{12}[\Gamma\right. & \left.: \Gamma_{0}(N / p)\right]-\frac{1}{2} \alpha_{2}(N / p, k p) \\
& -\frac{1}{3} \alpha_{3}(N / p, k p)-\epsilon_{\infty}\left(\frac{N}{p}\right)+1 .
\end{aligned}
$$

Considered asymptotically with respect to $N$, this interval has length $\frac{-k+p+1}{12}[\Gamma$ : $\left.\Gamma_{0}(N / p)\right]$. So the result of Theorem 1.4 is optimized when $p$ is as close to $k$ as possible. For example, when $k=4$ and $p=5$, then the length of the interval (1.4) is asymptotically one-third the length of the interval in (1.1).

The proofs of these results use techniques similar to those in [8, 7]. In order to prove these theorems, we will establish the analogous results in characteristic $p$. In particular, we give an improvement of a well-known theorem of Sturm on the maximal order of vanishing of a modular form in characteristic $p$. The tools involve facts on the integrality of modular forms, a modification of Sturm's original result to account for forced vanishing at the elliptic points, the trace map, the theory of modular forms $\bmod p$, and a recent result of Kilbourn [6, which, extending results of Deligne-Rapoport 3 and Weissauer [14, gives bounds for the $p$-adic valuation of the image of a cusp form $f \in S_{k}\left(\Gamma_{0}(N)\right)$ under the Atkin-Lehner operator $W_{p}^{N}$. We begin in the next section by stating the characteristic $p$ results and deducing from them Theorems 1.3 and 1.4 The following sections contain background material, the proof of the characteristic $p$ results, and examples.

\section{A result modulo $p$}

If $f(z)=\sum_{n=0}^{\infty} a(n) q^{n} \in M_{k}\left(\Gamma_{0}(N)\right) \cap \mathbb{Q}[[q]]$ and $p$ is prime, then define

$$
v_{p}(f):=\inf \left\{v_{p}(a(n)\}\right.
$$

(this infimum exists by the principle of bounded denominators). If $p$ is prime, then let $\mathbb{Z}_{(p)}$ denote the ring of $p$-integral rational numbers. If $f \in \mathbb{Z}_{(p)}[[q]]$, then we write $\bar{f}$ for its (coefficientwise) reduction modulo $p$, and if $v_{p}(f)=0$, then we denote by $\operatorname{ord}_{\infty}(\bar{f})$ the index of the first coefficient which does not vanish modulo $p$.

A well-known theorem of Sturm [13] gives bounds for the maximal order of vanishing of a modular form modulo $p$. Theorems 1.3 and 1.4 will follow from the next results, which improve Sturm's theorem in the cases under consideration.

Theorem 2.1. Suppose that $k \geq 2$ is an even integer, that $p \geq k+3$ is prime, and that $N$ is a positive integer with $p \| N$. Suppose that $f(z) \in M_{k}\left(\Gamma_{0}(N)\right) \cap \mathbb{Z}_{(p)}[[q]]$ and that $f \not \equiv 0(\bmod p)$. Then

$$
\operatorname{ord}_{\infty}(\bar{f}) \leq \frac{k p}{12} \cdot\left[\Gamma: \Gamma_{0}(N / p)\right]-\frac{1}{2} \alpha_{2}(N / p, k p)-\frac{1}{3} \alpha_{3}(N / p, k p) .
$$

Theorem 2.2. Suppose that $k \geq 2$ is an even integer, that $p \geq \max (5, k+1)$ is prime, and that $N$ is a positive integer with $p \| N$. Suppose that $f(z) \in S_{k}\left(\Gamma_{0}(N)\right) \cap$ $\mathbb{Z}_{(p)}[[q]]$ and that $f \not \equiv 0(\bmod p)$. Then

$$
\operatorname{ord}_{\infty}(\bar{f}) \leq \frac{k p}{12} \cdot\left[\Gamma: \Gamma_{0}(N / p)\right]-\frac{1}{2} \alpha_{2}(N / p, k p)-\frac{1}{3} \alpha_{3}(N / p, k p)-\epsilon_{\infty}(N / p)+1 .
$$


To deduce Theorem 1.3, we argue as follows. It suffices to prove the result for the form $f_{d}$ in the basis (1.2). Assume without loss of generality that $v_{p}\left(f_{d}\right)=0$. Since $\operatorname{ord}_{\infty}\left(f_{d}\right) \leq \operatorname{ord}_{\infty}\left(\bar{f}_{d}\right)$, Theorem 1.3 follows. Theorem 1.4 follows in the same manner.

\section{Preliminaries}

We first recall the values of some of the quantities introduced in the first section. A good reference is the table on page 107 of the book of Diamond and Shurman 4. We have

$$
\left[\Gamma: \Gamma_{0}(N)\right]=N \prod_{p \mid N}\left(1+\frac{1}{p}\right)
$$

The number of cusps on $X_{0}(N)$ is given by

$$
\epsilon_{\infty}(N)=\sum_{d \mid N} \phi(\operatorname{gcd}(d, N / d)) .
$$

Let $\epsilon_{2}(N), \epsilon_{3}(N)$ denote the numbers of elliptic points of orders 2 and 3 on $X_{0}(N)$, respectively. Then we have

$$
\begin{aligned}
& \epsilon_{2}(N)= \begin{cases}0 & \text { if } 4 \mid N, \\
\prod_{p \mid N}\left(1+\left(\frac{-4}{p}\right)\right) & \text { otherwise, }\end{cases} \\
& \epsilon_{3}(N)= \begin{cases}0 & \text { if } 9 \mid N, \\
\prod_{p \mid N}\left(1+\left(\frac{-3}{p}\right)\right) & \text { otherwise. }\end{cases}
\end{aligned}
$$

If $\alpha_{2}(N, k)$ and $\alpha_{3}(N, k)$ count the number of forced complex zeroes of a form $f \in M_{k}\left(\Gamma_{0}(N)\right)$ at the elliptic points of order 2 and order 3 , respectively, then

$$
\left(\alpha_{2}(N, k), \alpha_{3}(N, k)\right):=\left\{\begin{array}{lll}
\left(\epsilon_{2}(N), 2 \epsilon_{3}(N)\right) & \text { if } k \equiv 2 \quad(\bmod 12), \\
\left(0, \epsilon_{3}(N)\right) & \text { if } k \equiv 4 \quad(\bmod 12), \\
\left(\epsilon_{2}(N), 0\right) & \text { if } k \equiv 6 \quad(\bmod 12), \\
\left(0,2 \epsilon_{3}(N)\right) & \text { if } k \equiv 8 \quad(\bmod 12), \\
\left(\epsilon_{2}(N), \epsilon_{3}(N)\right) & \text { if } k \equiv 10 \quad(\bmod 12), \\
(0,0) & \text { if } k \equiv 0 \quad(\bmod 12) .
\end{array}\right.
$$

Then the quantity $\alpha(N, k)$ used in the introduction is given by

$$
\alpha(N, k):=\frac{1}{2} \alpha_{2}(N, k)+\frac{1}{3} \alpha_{3}(N, k) .
$$

We next recall some basic operators (a good reference is [2]). For any prime $p$, we define the linear operators $U_{p}$ and $V_{p}$ on Fourier expansions by

$$
\begin{aligned}
& \left(\sum a(n) q^{n}\right) \mid U_{p}:=\sum a(p n) q^{n}, \\
& \left(\sum a(n) q^{n}\right) \mid V_{p}:=\sum a(n) q^{p n} .
\end{aligned}
$$


We will always assume that $p$ is a prime with $p \| N$. For such primes, we define the Atkin-Lehner involution $W_{p}^{N}$ on $M_{k}\left(\Gamma_{0}(N)\right)$ by

$$
\left.f\right|_{k} W_{p}^{N}:=\left.f\right|_{k}\left(\begin{array}{cc}
p a & 1 \\
N b & p
\end{array}\right)
$$

where $a, b \in \mathbb{Z}$ and $p^{2} a-N b=p$. We then have

$$
\left.f\right|_{k} W_{p}^{N}=p^{\frac{k}{2}} f \mid V_{p} \quad \text { for } f \in M_{k}\left(\Gamma_{0}(N / p)\right) .
$$

We recall also the trace operator

$$
\operatorname{Tr}_{N / p}^{N}: M_{k}\left(\Gamma_{0}(N)\right) \rightarrow M_{k}\left(\Gamma_{0}(N / p)\right)
$$

defined by

$$
\operatorname{Tr}_{N / p}^{N}(f):=f+\left.p^{1-\frac{k}{2}} f\right|_{k} W_{p}^{N} \mid U_{p}
$$

The trace takes cusp forms to cusp forms. Finally, we define the familiar modular form

$$
E_{p-1}^{*}:=E_{p-1}-p^{p-1} E_{p-1} \mid V_{p} \in M_{p-1}\left(\Gamma_{0}(p)\right) .
$$

We have $E_{p-1}^{*} \equiv 1(\bmod p)$ and

$$
\left.E_{p-1}^{*}\right|_{p-1} W_{p}^{N} \equiv 0 \quad\left(\bmod p^{\frac{p+1}{2}}\right) \quad \text { for all } N \text { with } p \| N .
$$

We will make use of the following recent result of Kilbourn [6]. This generalizes the result of Weissauer [14] in the case of weight 2.

Theorem 3.1 (Kilbourn). Suppose that $f \in S_{k}\left(\Gamma_{0}(N)\right) \cap \mathbb{Q}[[q]]$ and that $p$ is a prime with $p \| N$ and $p \geq \max (5, k+1)$. Then $\left|v_{p}\left(\left.f\right|_{k} W_{p}^{N}\right)-v_{p}(f)\right| \leq \frac{k}{2}$.

We also require a minor modification of this theorem for modular forms.

Theorem 3.2. Suppose that $f \in M_{k}\left(\Gamma_{0}(N)\right) \cap \mathbb{Q}[[q]]$ and that $p$ is a prime with $p \| N$ and $p \geq k+3$. Then $\left|v_{p}\left(\left.f\right|_{k} W_{p}^{N}\right)-v_{p}(f)\right| \leq \frac{k}{2}$.

In the case of prime level, this result is proven in 3, Proposition 3.20. For the convenience of the reader, we will sketch Kilbourn's method as applied to Theorem 3.2. We seek a contradiction from the assumption (made without loss of generality after renormalization) that $f \in M_{k}\left(\Gamma_{0}(N)\right) \cap \mathbb{Z}_{(p)}[[q]]$ has $v_{p}(f)=0$ and $v_{p}\left(\left.f\right|_{k} W_{p}^{N}\right) \geq k / 2+1$. Defining $h:=\operatorname{Tr}_{N / p}^{N}(f) \in M_{k}\left(\Gamma_{0}(N / p)\right)$, we find from (3.7) that $h \equiv f\left(\bmod p^{2}\right)$. Let $m:=v_{p}(h-f) \geq 2$ and define $g:=$ $(h-f) / p^{m} \in M_{k}\left(\Gamma_{0}(N)\right) \cap \mathbb{Z}_{(p)}[[q]]$. Using the hypotheses and (3.6), it can be shown that $h\left|V_{p} \equiv p^{m-k / 2} g\right|_{k} W_{p}^{N}(\bmod p)$. Defining

$$
h^{\prime}:=\operatorname{Tr}_{N / p}^{N}\left(p^{m-k / 2}\left(\left.g\right|_{k} W_{p}^{N}\right)\left(E_{p-1}^{*}\right)^{k-2}\right) \in M_{(k-2) p+2}\left(\Gamma_{0}(N / p)\right),
$$

we find after a computation that $h^{\prime} \equiv h \mid V_{p}(\bmod p)$.

If $F \in M_{k}\left(\Gamma_{0}(N / p)\right) \cap \mathbb{Z}_{(p)}[[q]]$, define $\omega(F)=\inf \left\{k:\right.$ there exists $G \in M_{k}\left(\Gamma_{0}(N / p)\right) \cap \mathbb{Z}_{(p)}[[q]]$ with $\left.F \equiv G \quad(\bmod p)\right\}$.

The theory of modular forms modulo $p$ (see Section 4 of [5]) implies that $\omega\left(h^{\prime}\right)=$ $\omega\left(h^{p}\right)=p \omega(h)$. Since $k \leq p-3$ and $h$ is not identically zero, it follows that $\omega\left(h^{\prime}\right)=p k$, contradicting (3.9). 


\section{Proof of Theorem 2.1}

We require a slight sharpening of Sturm's theorem [13]. We follow Sturm's proof, but take account of forced vanishing at the elliptic points.

Theorem 4.1. Suppose that $k \geq 2$ is an even integer and that $N$ is a positive integer. Suppose that $f(z) \in M_{k}\left(\Gamma_{0}(N)\right) \cap \mathbb{Z}_{(p)}[[q]]$ and that $f \not \equiv 0(\bmod p)$. Then

$$
\operatorname{ord}_{\infty}(\bar{f}) \leq \frac{k}{12} \cdot\left[\Gamma: \Gamma_{0}(N)\right]-\frac{1}{3} \alpha_{3}(N, k)-\frac{1}{2} \alpha_{2}(N, k) .
$$

If in fact $f(z) \in S_{k}\left(\Gamma_{0}(N)\right) \cap \mathbb{Z}_{(p)}[[q]]$, then

$$
\operatorname{ord}_{\infty}(\bar{f}) \leq \frac{k}{12} \cdot\left[\Gamma: \Gamma_{0}(N)\right]-\frac{1}{3} \alpha_{3}(N, k)-\frac{1}{2} \alpha_{2}(N, k)-\epsilon_{\infty}(N)+1 .
$$

Proof. Define $m:=\left[\Gamma: \Gamma_{0}(N)\right]$ and let $\gamma_{v}, v=1, \ldots, m$ (where $\gamma_{1}$ is the identity) be the representatives of $\Gamma \backslash \Gamma_{0}(N)$. Following Sturm's argument, we fix a number field $K$ containing the coefficients of each form $\left.f\right|_{k} \gamma_{v}$, and denote by $\mathcal{O}$ the ring of integers of $K$. Let $\lambda$ be any place above $p$. For each $v$, we find $A_{v} \in K^{\times}$such that $v_{\lambda}\left(\left.A_{v} f\right|_{k} \gamma_{v}\right)=0$ and consider the form

$$
G:=\left.f \prod_{v=2}^{m} A_{v} f\right|_{k} \gamma_{v} \in S_{k m}(\Gamma)
$$

Note that $G \not \equiv 0(\bmod \lambda)$. For $h=2,3$, we see that for each complex zero of $f$ at an elliptic fixed point of order $h$ on a fundamental domain for $\Gamma_{0}(N)$, the function $G$ has precisely one zero at an elliptic fixed point of order $h$ on a fundamental domain for $\Gamma$. Since $E_{4}$ and $E_{6}$ have simple zeros at the points of orders 3,2 for $\Gamma$, we conclude that

$$
G^{\prime}:=\frac{G}{E_{4}^{\alpha_{3}(N, k)} E_{6}^{\alpha_{2}(N, k)}} \in S_{k m-4 \alpha_{3}(N, k)-6 \alpha_{2}(N, k)}(\Gamma) .
$$

Since $f$ is a cusp form, we see that for each $v \geq 2$, we have an expansion of the form

$$
\left.A_{v} f\right|_{k} \gamma_{v}=c_{v} q^{1 / h_{v}}+\cdots,
$$

where $h_{v}$ is the width of the cusp corresponding to $\gamma_{v}$. Since each such cusp corresponds to exactly $h_{v}$ of the elements $\gamma_{v}$, we conclude that $G^{\prime}$ vanishes $\bmod \lambda$ at $\infty$ to order at least

$$
\operatorname{ord}_{\lambda}(\bar{f})+\epsilon_{\infty}(N)-1
$$

By Sturm's result in level one, this quantity is at most one-twelfth the weight of $G^{\prime}$, which gives the theorem for cusp forms. The proof for modular forms is the same.

We will now prove the following.

Theorem 4.2. Suppose that $N$ and $k$ are positive integers and that $p \geq 5$ is a prime with $p \| N$. Suppose that $f \in M_{k}\left(\Gamma_{0}(N)\right) \cap \mathbb{Z}_{(p)}[[q]]$ has

$$
v_{p}(f)=0, \quad v_{p}\left(\left.f\right|_{k} W_{p}^{N}\right) \geq a .
$$


(1) If $p \geq k+3$, then

$$
\begin{aligned}
\operatorname{ord}_{\infty}(\bar{f}) \leq & \frac{k+\left(\frac{k}{2}-a\right)(p-1)}{12}\left[\Gamma: \Gamma_{0}(N / p)\right] \\
& -\frac{1}{3} \alpha_{2}\left(N / p, k+\left(\frac{k}{2}-a\right)(p-1)\right)-\frac{1}{2} \alpha_{3}\left(N / p, k+\left(\frac{k}{2}-a\right)(p-1)\right) .
\end{aligned}
$$

(2) If $p \geq k+1$ and $f \in S_{k}\left(\Gamma_{0}(N)\right)$, then

$$
\begin{aligned}
& \operatorname{ord}_{\infty}(\bar{f}) \leq \frac{k+\left(\frac{k}{2}-a\right)(p-1)}{12}\left[\Gamma: \Gamma_{0}(N / p)\right] \\
- & \frac{1}{3} \alpha_{2}\left(N / p, k+\left(\frac{k}{2}-a\right)(p-1)\right)-\frac{1}{2} \alpha_{3}\left(N / p, k+\left(\frac{k}{2}-a\right)(p-1)\right)-\epsilon_{\infty}(N / p)+1 .
\end{aligned}
$$

Theorems 2.1 and 2.2 follow immediately since by Theorems 3.1 and 3.2 we have $a \geq-k / 2$ in each case.

Proof of Theorem 4.2, Let $f$ be as in the hypotheses of the first part. Then consider the form

$$
\begin{aligned}
F & :=\operatorname{Tr}_{N / p}^{N}\left(f\left(E_{p-1}^{*}\right)^{\frac{k}{2}-a}\right) \\
& =f\left(E_{p-1}^{*}\right)^{\frac{k}{2}-a}+p^{1-\frac{k+\left(\frac{k}{2}-a\right)(p-1)}{2}}\left(\left.\left.f\right|_{k} W_{p}^{N} \cdot\left(E_{p-1}^{*}\right)^{\frac{k}{2}-a}\right|_{(p-1)\left(\frac{k}{2}-a\right)} W_{p}^{N}\right) \mid U_{p} .
\end{aligned}
$$

Then $F \in M_{k+\left(\frac{k}{2}-a\right)(p-1)}\left(\Gamma_{0}(N / p)\right) \cap \mathbb{Q}[[q]]$. Moreover, a computation using (3.8) shows that we have $F \equiv f(\bmod p)$. By Theorem 4.1 we conclude that

$$
\begin{aligned}
\operatorname{ord}_{\infty}(\bar{f})= & \operatorname{ord}_{\infty}(\bar{F}) \leq \frac{k+\left(\frac{k}{2}-a\right)(p-1)}{12}\left[\Gamma: \Gamma_{0}(N / p)\right] \\
& -\frac{1}{3} \alpha_{2}\left(N / p, k+\left(\frac{k}{2}-a\right)(p-1)\right)-\frac{1}{2} \alpha_{3}\left(N / p, k+\left(\frac{k}{2}-a\right)(p-1)\right) .
\end{aligned}
$$

The second assertion follows in a similar manner.

Finally, we prove Theorem 1.1. If $f \in S_{2}\left(\Gamma_{0}(N)\right)$ is as in the hypotheses, then, taking $k=2$ and $a=0$ in Theorem 4.2. we find that

$\operatorname{ord}_{\infty}(\bar{f}) \leq \frac{p+1}{12}\left[\Gamma: \Gamma_{0}(N / p)\right]-\epsilon_{\infty}(N / p)-\frac{1}{2} \alpha_{2}(N / p, p+1)-\frac{1}{3} \alpha_{3}(N / p, p+1)+1$.

A computation shows that we have $\alpha_{2}(N / p, p+1)=\frac{1}{2} \epsilon_{2}(N), \alpha_{3}(N / p, p+1)=$ $\epsilon_{3}(N)$, and $\epsilon_{\infty}(N / p)=\frac{1}{2} \epsilon_{\infty}(N)$. Thus,

$$
\operatorname{ord}_{\infty}(\bar{f}) \leq \frac{\left[\Gamma: \Gamma_{0}(N)\right]}{12}-\frac{1}{2} \epsilon_{\infty}(N)-\frac{1}{4} \epsilon_{2}(N)-\frac{1}{3} \epsilon_{3}(N)+1 .
$$

The right-hand side is precisely the genus of $X_{0}(N)$, which proves Theorem 1.1.

Corollary 1.2 can be checked explicitly when $p=2,3$. For other primes, we note that if $g(N / p)=0$ and $f \in S_{2}\left(\Gamma_{0}(N)\right) \cap \mathbb{Z}_{(p)}[[q]]$, then $\operatorname{Tr}_{N / p}^{N}\left(f \mid W_{p}^{N}\right)=0$, so that we must have $v_{p}\left(f \mid W_{p}^{N}\right)=v_{p}\left(f \mid U_{p}\right) \geq 0$.

\section{EXAMPLES}

We provide more examples of spaces for which Theorems 1.3 and 1.4 are sharp. Let $N^{\prime} \geq 1$ be a squarefree integer. Define the form

$$
f_{N^{\prime}}(z):=\left(\prod_{d \mid N^{\prime}} \eta(d z)^{\mu\left(N^{\prime} / d\right) d}\right)^{\alpha},
$$


where $\mu(n)$ is the Möbius function and

$$
\alpha:= \begin{cases}24 & \text { if } N^{\prime}=1 \\ 8 & \text { if } N^{\prime}=2 \\ 6 & \text { if } N^{\prime}=3 \\ 2 & \text { if } N^{\prime}=6, p, \text { or } 2 p \text { where } p \geq 5 \text { is prime } \\ 1 & \text { otherwise. }\end{cases}
$$

Set

$$
k:=\frac{\alpha \phi\left(N^{\prime}\right)}{2} .
$$

Using standard criteria (a convenient reference is Section 1.4 of [1]) one can check that $f_{N^{\prime}}(z) \in M_{k}\left(\Gamma_{0}\left(N^{\prime}\right)\right)$ and that

$$
\operatorname{ord}_{\infty}\left(f_{N^{\prime}}(z)\right)=\frac{\alpha \phi\left(N^{\prime}\right) \sigma_{1}\left(N^{\prime}\right)}{24}=\frac{k}{12}\left[\Gamma: \Gamma_{0}\left(N^{\prime}\right)\right] .
$$

If $N^{\prime}$ is not squarefree, then write $N^{\prime}=N_{1} N_{2}$ where $N_{1}$ is the largest squarefree divisor of $N^{\prime}$. Then define the form $f_{N^{\prime}}(z):=f_{N_{1}}(z) \mid V_{N_{2}} \in M_{\alpha \phi\left(N_{1}\right) / 2}\left(\Gamma_{0}\left(N^{\prime}\right)\right)$.

For all $N^{\prime} \geq 1$ it follows that

$$
\operatorname{ord}_{\infty}\left(f_{N^{\prime}}(z)\right)=\frac{k}{12}\left[\Gamma: \Gamma_{0}\left(N^{\prime}\right)\right] .
$$

If $p \geq k+3$, let $N=p N^{\prime}$. Theorem 1.3 asserts that each non-zero form $f \in$ $M_{k}\left(\Gamma_{0}(N)\right)$ has

$$
\operatorname{ord}_{\infty}(f) \leq \frac{k p}{12}\left[\Gamma: \Gamma_{0}(N)\right] .
$$

We see from (5.1) that equality holds in (5.2) for the form $f_{N^{\prime}}(p z) \in M_{k}\left(\Gamma_{0}(N)\right)$. Therefore Theorem 1.3 is sharp for these spaces.

We turn to Theorem 1.4 Suppose that $k \geq 2$, and that $p \geq 12 k+1$ is prime. Then the order of vanishing of the form $F(z):=\Delta(p z)^{k}=q^{k p}+\cdots \in S_{12 k}\left(\Gamma_{0}(p)\right)$ agrees with the upper bound provided by Theorem 1.4 .

There are other examples where Theorem 1.4 is sharp. For example, define

$$
F(z):=\frac{\eta(6 z) \eta(9 z) \eta^{6}(21 z) \eta^{34}(126 z)}{\eta^{2}(18 z) \eta^{11}(42 z) \eta^{17}(63 z)} \in S_{6}\left(\Gamma_{0}(126)\right) .
$$

Then we have $\alpha_{2}(18,42) / 2=\alpha_{3}(18,42) / 3=0$ and

$$
\operatorname{ord}_{\infty}(F)=119=\frac{6 \cdot 7}{12}\left[\Gamma: \Gamma_{0}(18)\right]-\epsilon_{\infty}(18)+1 .
$$

Another example is provided by the form

$$
F=2 q^{99}+2 q^{101}-3 q^{104}+\cdots \in S_{6}\left(\Gamma_{0}(175)\right) .
$$

Then, $\alpha_{2}(25,42) / 2=1, \alpha_{3}(25,42) / 3=0$ and

$$
\operatorname{ord}_{\infty}(F)=99=\frac{6 \cdot 7}{12}\left[\Gamma: \Gamma_{0}(25)\right]-\alpha_{2}(25,42) / 2-\alpha_{3}(25,42) / 3-\epsilon_{\infty}(25)+1 .
$$

In closing, we mention several other forms for which equality holds in Theorem 1.4 (there are other examples of the same sort). For example, this occurs for 
the following forms:

$$
\begin{aligned}
& \frac{\eta(z) \eta^{13}(77 z)}{\eta(7 z) \eta(11 z)} \in S_{6}\left(\Gamma_{0}(77)\right), \\
& \frac{\eta^{30}(44 z) \eta^{2}(2 z)}{\eta^{2}(4 z) \eta^{14}(22 z)} \in S_{8}\left(\Gamma_{0}(44)\right), \\
& \frac{\eta^{29}(99 z) \eta(3 z)}{\eta^{9}(33 z) \eta(9 z)} \in S_{10}\left(\Gamma_{0}(99)\right), \\
& \frac{\eta^{47}(46 z) \eta(z)}{\eta^{23}(23 z) \eta(2 z)} \in S_{12}\left(\Gamma_{0}(46)\right)
\end{aligned}
$$

\section{REFERENCES}

1. A. O. L. Atkin, Weierstrass points at cusps of $\Gamma_{0}(n)$, Ann. of Math. (2) 85 (1967), 42-45. MR 0218561 (36:1646)

2. A. O. L. Atkin and J. Lehner, Hecke operators on $\Gamma_{0}(m)$, Math. Ann. 185 (1970), 134-160. MR.0268123 (42:3022)

3. P. Deligne and M. Rapoport, Les schémas de modules de courbes elliptiques, Modular functions of one variable, II (Proc. Internat. Summer School, Univ. Antwerp, Antwerp, 1972), Lecture Notes in Math., vol. 349. Springer, Berlin, 1973, pp. 143-316. MR0337993 (49:2762)

4. Fred Diamond and Jerry Shurman, A first course in modular forms, Graduate Texts in Mathematics, vol. 228, Springer-Verlag, New York, 2005. MR2112196 (2006f:11045)

5. Benedict H. Gross, A tameness criterion for Galois representations associated to modular forms $(\bmod p)$, Duke Math. J. 61 (1990), no. 2, 445-517. MR.1074305 (91i:11060)

6. Timothy Kilbourn, Congruence properties of Fourier coefficients of modular forms, preprint.

7. Kilian Kilger, Weierstrass points on $X_{0}(p \ell)$ and arithmetic properties of Fourier coffficients of cusp forms, Ramanujan J. 17 (2008), 321-330.

8. Winfried Kohnen, A short remark on Weierstrass points at infinity on $X_{0}(N)$, Monatsh. Math. 143 (2004), no. 2, 163-167. MR2097501 (2005k:11119)

9. J. Lehner and M. Newman, Weierstrass points of $\Gamma_{0}(n)$, Ann. of Math. (2) 79 (1964), 360368. MR0161841(28:5045)

10. A. P. Ogg, On the Weierstrass points of $X_{0}(N)$, Illinois J. Math. 22 (1978), no. 1, 31-35. MR 0463178 (57:3136)

11. Ken Ono, The web of modularity: Arithmetic of the coefficients of modular forms and q-series, CBMS Regional Conference Series in Mathematics, vol. 102, published for the Conference Board of the Mathematical Sciences, Washington, DC, by the Amer. Math. Soc., Providence, RI, 2004. MR2020489 (2005c:11053)

12. Bruno Schoeneberg, Elliptic modular functions: An introduction, translated from the German by J. R. Smart and E. A. Schwandt, Die Grundlehren der Mathematischen Wissenschaften, Band 203, Springer-Verlag, New York, 1974. MR0412107 (54:236)

13. Jacob Sturm, On the congruence of modular forms, Number Theory (New York, 1984-1985), Lecture Notes in Math., vol. 1240, Springer, Berlin, 1987, pp. 275-280. MR894516 (88h:11031)

14. R. Weissauer, Integral forms on $\Gamma_{0}(p n)$, preprint (2002).

Department of Mathematics, University of Illinois, Urbana, Illinois 61801

E-mail address: ahlgren@math.uiuc.edu

Department of Mathematics, University of Illinois, Urbana, Illinois 61801

E-mail address: nmasri@math.uiuc.edu

Department of Mathematics, University of Illinois, Urbana, Illinois 61801

E-mail address: jarouse@math.uiuc.edu 